Research Article

\title{
Piezoresistivity of Cement Matrix Composites Incorporating Multiwalled Carbon Nanotubes due to Moisture Variation
}

\author{
Yanfeng Wang, ${ }^{1,2}$ Xiaohua Zhao $\mathbb{D}^{1,3}$ and Yi Zhao ${ }^{2}$ \\ ${ }^{1}$ Department of Civil Engineering, Shantou University, Shantou 515063, China \\ ${ }^{2}$ Department of Civil Engineering and Architecture, Zhongyuan University of Technology, Zhengzhou, China \\ ${ }^{3}$ Key Laboratory of Structure and Wind Tunnel of Guangdong Higher Education Institutes, Shantou 515063, China \\ Correspondence should be addressed to Xiaohua Zhao; xhzhao@stu.edu.cn
}

Received 6 August 2019; Accepted 3 January 2020; Published 28 February 2020

Academic Editor: Yinshan Tang

Copyright (c) 2020 Yanfeng Wang et al. This is an open access article distributed under the Creative Commons Attribution License, which permits unrestricted use, distribution, and reproduction in any medium, provided the original work is properly cited.

Cementitious composites usually work under moisture condition. Presently, the piezoresistivity of cementitious composites incorporating multiwalled carbon nanotubes (MWCNTs) due to moisture variation was experimentally investigated. The variation of moisture content was controlled by drying specimens in an oven. In most cases of moisture content, the composites were observed to present positive piezoresistivity during the process of cyclic compression. While moisture content was in a specific range, the composites exhibited negative piezoresistivity. The whole transition from positive piezoresistivity to negative piezoresistivity and then positive piezoresistivity was obtained with moisture variation. Moreover, the amplitude of piezoresistivity changed in the process. These phenomena may be explained through the combination of ionic conduction and electronic conduction. A theoretical model of piezoresistivity, able to predict the effects of porosity, the volume fraction of MWCNTs, and the connectivity parameters, is proposed. Numerical results with the model show that the calculated piezoresistive responses of specimens agree reasonably well with testing data.

\section{Introduction}

The concept of incorporating nanofillers into cementitious materials in order to improve their performance has received considerable attention over the past decade $[1,2]$. In particular, carbon nanotubes (CNTs) have attracted significant interest because they have demonstrated numerous exceptional properties, such as high specific area ratio, high strength, high toughness, high conductivity, and low density [3-5]. Various studies in the literature have shown that the addition of an appropriate amount of CNTs can enhance the strength of cementitious materials and reduce the electric resistance of them [6-17]. More important, the cementitious composites containing CNTs are found to present piezoresistive behaviors.

Piezoresistivity is a kind of coupled electrical-mechanical property that the electric resistivity of a material changes with strain. If the electric resistivity decreases with compressive strain, piezoresistivity is defined to be positive.
Otherwise, piezoresistivity is negative. Due to this property, the cementitious composites containing CNTs are able to sense strain through the variation of electric resistance. This finding is of great importance for the nondestructive monitoring of structures in that the composite materials themselves can be used as stress/strain sensors and be used to monitor both the generation and propagation of damage, while being at the same time for structural functions.

Li et al. [18] firstly developed piezoresistive CNT/cement composites, using multiwalled carbon nanotubes (MWCNTs) treated by a mixed solution of $\mathrm{H}_{2} \mathrm{SO}_{4}$ and $\mathrm{HNO}_{3}$, and measured the piezoresistivity of the composites under uniaxial compression. Saafi [19] studied the piezoresistivity of the cementitious composites containing single-walled CNTs and demonstrated that this piezoresistive behavior is able to detect damage in concrete structures. Han et al. [20] further investigated the possibility of this piezoresistive behavior for traffic monitoring. In addition, they [21] examined the effect of stress amplitudes and loading rates on the piezoresistivity. 
Experimental results indicated that the cementitious composites containing CNTs presented stable and reversible piezoresistive responses under various conditions. $\mathrm{Yu}$ and Kwon [22] also studied the piezoresistive property of the $\mathrm{CNT} /$ cement composite to explore its feasibility as an embedded stress sensor for civil structures such as roadways, levees, and bridges. The results show that the electrical resistance of the CNT/cement composite changes with the compressive stress level, indicating the potential of using the $\mathrm{CNT/cement} \mathrm{composite} \mathrm{as} \mathrm{a} \mathrm{stress} \mathrm{sensor} \mathrm{for} \mathrm{civil} \mathrm{structures.}$ Recently, Konsta-Gdoutos and Aza [23] explored the piezoresistivity of cementitious composites with the hybrid addition of both CNTs and carbon nanofibers. The tests showed that the composites under uniaxial compression are highly piezoresistive.

However, the cementitious composites containing CNTs are porous materials, and capillary water exists in cement matrix even if the hydration process has completed. Moreover, the cementitious composites usually work in a moisture condition. One concern then arises that the piezoresistivity may be affected by moisture content in the composites, as water provides ions that contribute to ionic conduction. This effect is relevant to the practical application of the composites as piezoresistive structural materials. Han et al. [24] tested the effect on the piezoresistive sensitivity by change moisture content in the composites. When the variation of water content was $0.1,1.3,3.3,5.7,7.6$, and $9.9 \%$, the stress sensitivity became $0.60,0.61,0.73,0.68,0.34$, and $0.06 \mathrm{k} \Omega / \mathrm{MPa}$, respectively. Kim et al. [25] investigated the piezoresistive behaviors of $\mathrm{CNT} /$ cement mortars with different water-binder ratios. They found that the variation of piezoresistive sensitivity and stability induced by moisture content decreased when the water-binder ratio was low.

These efforts highlight the strong relation of piezoresistivity with moisture content. Due to moisture, electric conduction in the composites will be induced by not only the electron transmission through the network of CNTs, but also ionic conduction through the network of capillary pores or voids. Under deformation, these two networks may change, leading to the variation of piezoresistive responses. This means that the piezoresistive behaviors are controlled by two kinds of mechanisms, namely, ionic conduction and electron transmission. Unfortunately, to the knowledge of the authors, the role each mechanism plays during deformation has not been well understood. Furthermore, a predictive theory of piezoresistivity, incorporating both mechanisms, is still not available.

With the above in mind, the effect of moisture content on the piezoresistive behaviors of cementitious composites containing MWCNTs is presently considered. Five tests were performed for the composites with variable moisture content. In order to explore the effect of ionic conduction, the plain cementitious composites were also tested. Different from the results previously reported, both positive and negative piezoresistive behaviors were observed during the testing. A possible reason for the behaviors considering both ionic conduction and electron transmission is discussed. Based on this, a predictive theory of piezoresistivity is proposed and compared with test results.

\section{Experimental Methods}

2.1. Materials. The cement-based mortars were prepared with ordinary Portland cement (42.5), and the fine aggregate was natural sand (in accordance with China ISO Standard). The ratio of water/cement/sand was $0.4: 1: 1.5$. The MWCNTs used were supplied by Chengdu Organic Chemicals Company, China. Their properties are shown in Table 1. The surfactant for dispersing the MWCNTs was polyvinylpyrrolidone (PVP), as provided by $\mathrm{Xi}$ Long Company of Shantou, China.

2.2. Preparation of Specimens. Three mixes of cement-based mortars were fabricated for the piezoresistivity test. Table 2 shows the proportions of these composites. The amount of MWCNTs added was $0.1 \%$ and $0.3 \%$ by weight of cement, respectively. These additions were under the percolation threshold value of cementitious composites containing MWCNTs. Three specimens were prepared for each mix.

To disperse MWCNTs uniformly, surfactant (PVP) was firstly mixed with water and stirred for one minute by hand. MWCNTs were then added into this aqueous solution and sonicated with an ultrasonicator (KQ 218 Ultrasonic generator) for 40 minutes. A rotary mixer with a flat beater was used to mix this solution and cement for about 3 minutes. Finally, fine sands were added. All the ingredients were mixed for another 3 minutes.

After pouring the mixes into oiled molds $(40 \times 40 \times 160 \mathrm{~mm})$, an electric vibrator was used to facilitate compaction and decrease the amount of air bubbles. During the process, four pieces of stainless metallic grids $(50 \times 10 \times 2 \mathrm{~mm})$ were embedded in each of the specimens for the purpose of electrical resistance measurement by the four-probe method, as shown in Figure 1. The two outer pieces $(100 \mathrm{~mm}$ apart, symmetrically positioned relative to the center plane perpendicular to the longitudinal direction) were for passing current, while the two inner pieces $(60 \mathrm{~mm}$ apart, symmetrically positioned relative to the center plane perpendicular to the longitudinal direction) were for voltage measurement.

One day after casting, all specimens were demolded and cured in a standard curing box with a constant temperature of $22^{\circ} \mathrm{C}$ and the relative humidity above $95 \%$ until the time of testing.

2.3. Resistance and Piezoresistivity Measurement. The first test for the piezoresistivity of these composites began at the age of 28 days. The test setup is shown in Figure 2. Before testing, resistance was continuously measured till it became stabilized. Then, a compressive testing was performed under repeated loads. Five loading cycles were carried out and all stress amplitudes were controlled within the elastic regime. The loads were applied on the two ends in the longitudinal direction of specimens. Both strain and resistance were measured during the loading process. The longitudinal strain was measured by using a strain gage that had been adhesively attached to the surface along the length of each specimen. Resistance was measured using a multimeter 
TABle 1: Properties of carboxyl MWCNTs.

Outside diameter $(\mathrm{nm})$ Length $(\mu \mathrm{m}) \quad W(\mathrm{ash})(\%)-\mathrm{COOH}(\%) \quad$ Purity (\%) $\quad$ Specific surface area $\left(\mathrm{m}^{2} / \mathrm{g}\right) \quad$ Electric conductivity $(\mathrm{s} / \mathrm{cm})$

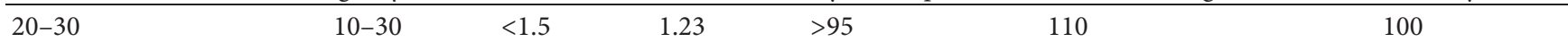

TABle 2: Mix proportions of MWCNTs reinforced cement mortars ${ }^{\mathrm{a}}$.

\begin{tabular}{lccccc}
\hline Mix & Cement & Water & Sand & MWCNTs (\%) & PVP (\%) \\
\hline CM & 1 & 0.4 & 1.5 & 0.00 & 0.04 \\
WCM1 & 1 & 0.4 & 1.5 & 0.10 & 0.04 \\
WCM3 & 1 & 0.4 & 1.5 & 0.30 & 0.09 \\
\hline
\end{tabular}

${ }^{\mathrm{a}}$ Given as percent of cement content by mass.

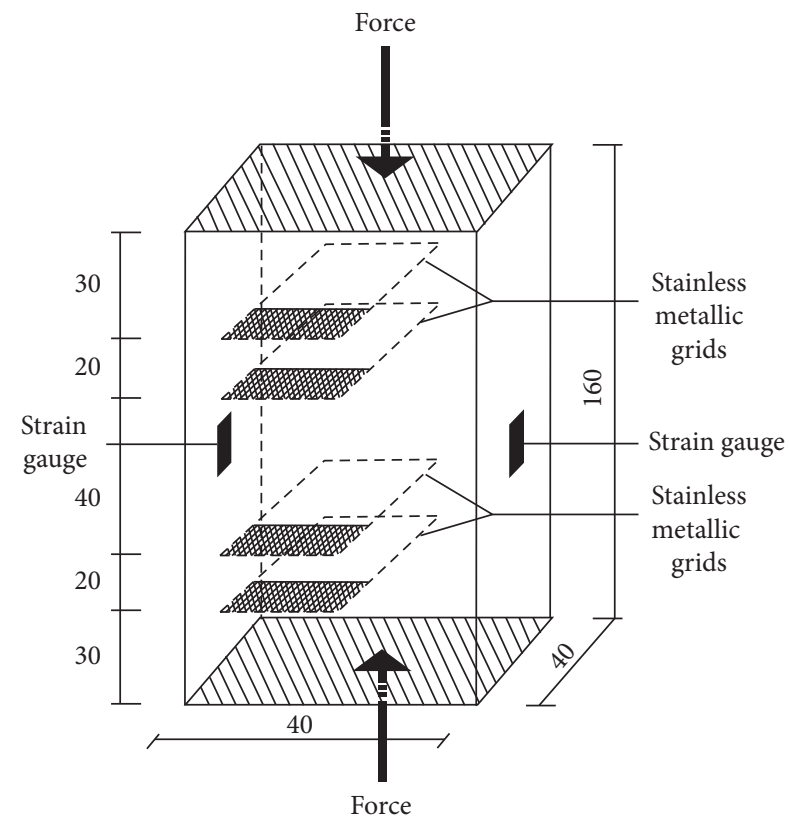

FIGURE 1: Sketch of the specimen.

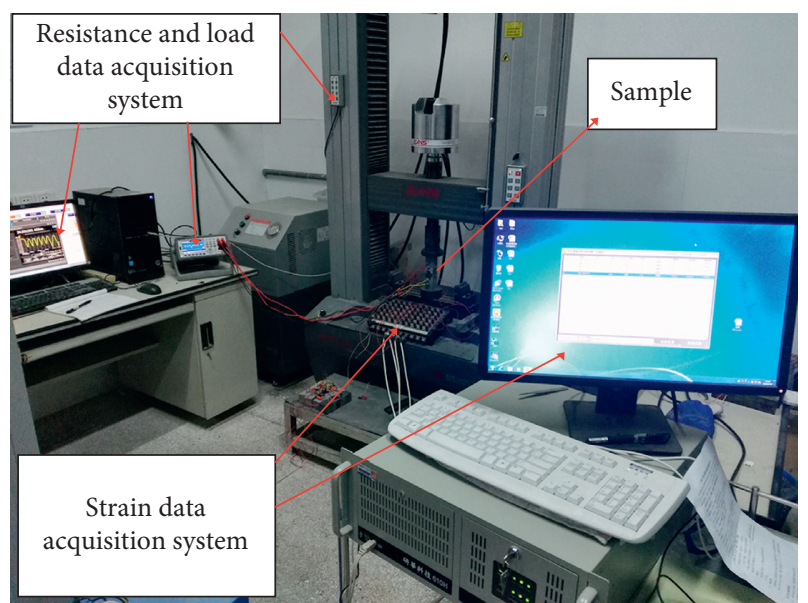

FIgURE 2: The piezoresistivity test under cyclic compressive loading.

(Agilent 34401A) and the four-probe method. All of the measurements were automatically recorded through a data logger. After the testing, in order to investigate the influence of moisture content on the piezoresistivity of the composites, all specimens were further dried in an oven for at least two days at a temperature of $50^{\circ} \mathrm{C}$ and the relative humidity of $30 \%$ till an obvious weight loss was observed. Thereafter, they were cooled for one day under room conditions and then tested for the second time. Following this procedure, another four tests were conducted. All specimens were weighed before each test.

The piezoresistivity of each composite was calculated by the following equation:

$$
\frac{\Delta R}{R_{0}}=\left(\frac{R-R_{0}}{R_{0}}\right) \times 100 \%,
$$

with $R$ being resistance and $R_{0}$ being the initial value prior to any loading.

2.4. Scanning Electron Microscope (SEM) and Porosity Analysis. The morphology and microstructure of the fractured surfaces of the composites were observed using a HITACHI SU 6600 scanning electron microscope at accelerating voltage of $2.0 \mathrm{kV}$. Three samples were prepared for each mix and kept in alcohol. They were dried in a vacuumdrying oven for $24 \mathrm{~h}$ and gold coated before examination.

The porosity analysis was also performed. An Auto Pore IV 9510 Mercury Intrusion Porosimetry (MIP), able to determine the distribution of pores from $3 \mathrm{~nm}$ to $1000 \mu \mathrm{m}$, was used for the measurement. The contact angle and mercury surface tension used were $130^{\circ}$ and $485 \mathrm{erg} / \mathrm{cm}^{2}$, respectively.

\section{Results and Discussion}

Table 3 shows the result of specimen porosity measured by MIP. The total porosity of CM reached the value of $28.79 \%$. Even though the addition of MWCNTs might produce a "filling void effect," the porosity of WCM1 and WCM3 remained $27.24 \%$ and $27.19 \%$, respectively. This indicates that there existed a large number of capillary pores and voids in the composites. Indeed, the SEM image in Figure 3 demonstrates the existence of these pores and voids. In a moisture condition, the pores and voids are usually filled with water, which will play an important role for the electric conduction in the composites $[25,26]$. On one side, the pore water may connect forming pathways of ionic conduction. On the other side, the pore water reduces the height of potential barrier between adjacent MWCNTs, increasing the transmission of electrons between them [27, 28].

Moisture in the composites changed after oven drying. Table 4 shows the average weight loss of three specimens before each piezoresistive test. It is seen that the weight loss of CM, WCM1, and WCM3 decreased monotonically from $0.75 \%$ to $3.29 \%$, from $0.67 \%$ to $3.36 \%$, and from $1.12 \%$ to $3.11 \%$, respectively. Clearly, the weight loss is attributed to 
TABle 3: Porosity of the specimens measured by MIP.

\begin{tabular}{lcccc}
\hline Mix & Total intruded volume $(\mathrm{ml} / \mathrm{g})$ & Average pore diameter $(\mathrm{nm})$ & Total porosity $(\%)$ & Total pore area $\left(\mathrm{m}^{2} / \mathrm{g}\right)$ \\
\hline CM & 0.165 & 21.57 & 28.79 & 30.72 \\
WCM1 & 0.155 & 19.8 & 27.24 & 31.29 \\
WCM3 & 0.146 & 19.74 & 27.19 & 29.76 \\
\hline
\end{tabular}

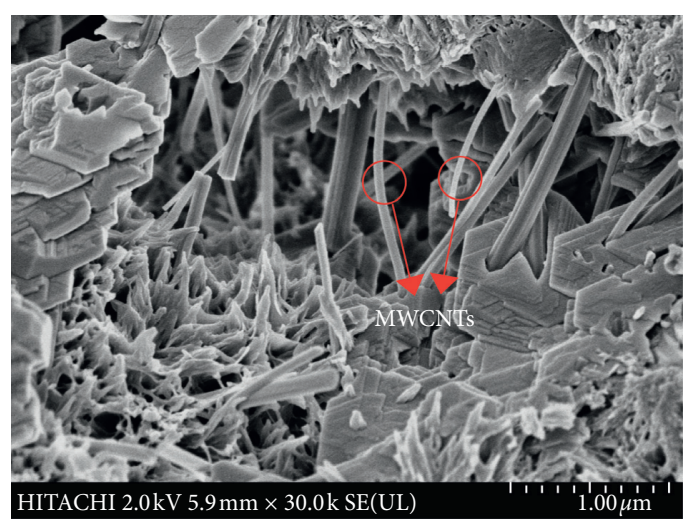

(a)

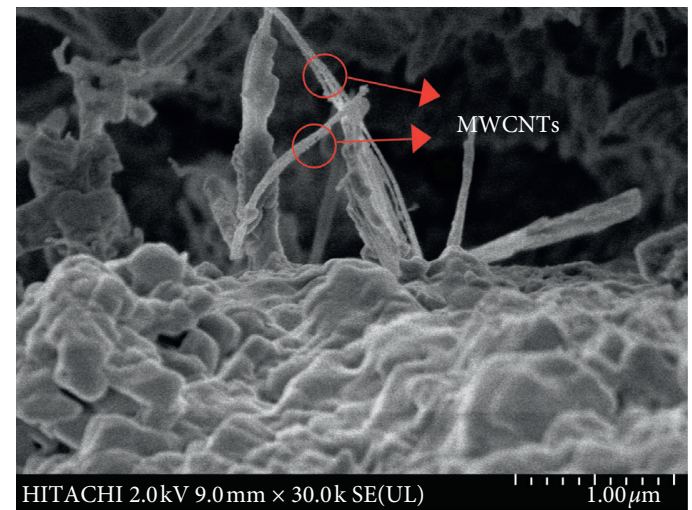

(b)

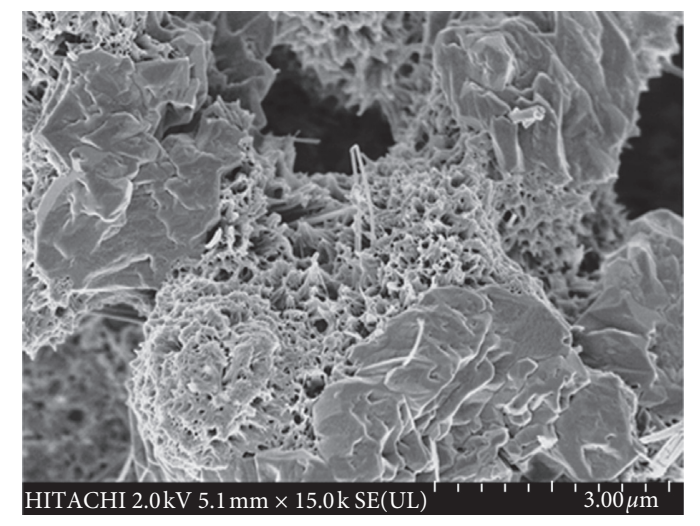

(c)

Figure 3: SEM image (a) WCM3, (b) WCM1, and (c) CM.

TABle 4: Average weight loss of specimens.

\begin{tabular}{lccccc}
\hline Test number & 1 & 2 & 3 & 4 & 5 \\
\hline Mass loss of CM & 0 & $0.75 \%$ & $1.57 \%$ & $2.18 \%$ & $3.29 \%$ \\
Mass loss of WCM1 & 0 & $0.67 \%$ & $1.58 \%$ & $2.25 \%$ & $3.36 \%$ \\
Mass loss of WCM3 & 0 & $1.12 \%$ & $1.91 \%$ & $2.54 \%$ & $3.11 \%$ \\
\hline
\end{tabular}

the reduction of moisture in the specimens, since no physical or chemical change took place in the drying process. Due to the moisture reduction, moisture content in the specimens decreased gradually with the increase of test numbers and was obviously different from one test to another.

Accordingly, piezoresistivity varied due to moisture content. As shown in Table 5, there appear two kinds of piezoresistivity in the five tests, which are positive piezoresistivity and negative piezoresistivity, respectively. The whole testing process saw the transition of piezoresistivity from positive to negative and then positive.

It was observed in the five tests that both WCM1 and WCM3 presented similar piezoresistive responses during
Table 5: Piezoresistivity for each test ( $\mathrm{P}=$ positive; $\mathrm{N}=$ negative).

\begin{tabular}{llllll}
\hline Test number & 1 & 2 & 3 & 4 & 5 \\
\hline Piezoresistivity & $\mathrm{P}$ & $\mathrm{N}$ & $\mathrm{P}$ & $\mathrm{P}$ & $\mathrm{P}$ \\
\hline
\end{tabular}

each test. Moreover, the piezoresistivity of three specimens in the same group exhibited a similar trend. Therefore, only results for WCM3 and CM are given in the following.

Figure 4 depicts the piezoresistive responses of specimens for the first test. In this case, moisture content was the highest. It is seen that both CM and WCM3 had piezoresistive behavior. During cyclic compression, the strain varied linearly up to the peak value about $0.3 \%$ and returned to zero at the end of each cycle of loading, indicating that the strain was within the elastic regime. In each cycle, the value of $\Delta R / R_{0}$ decreased monotonically upon the compressive loading, while increased monotonically upon unloading, exhibiting positive piezoresistivity.

The results suggest that the piezoresistive behavior of cementitious composites can be induced by the transmission 


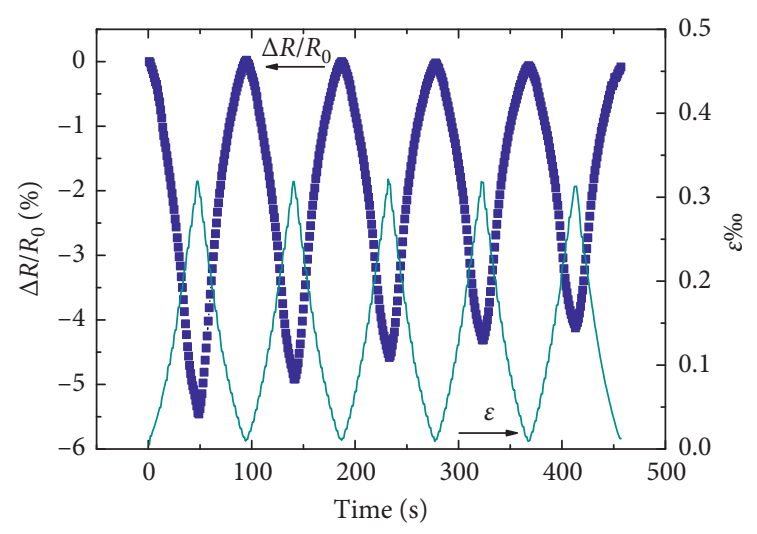

(a)

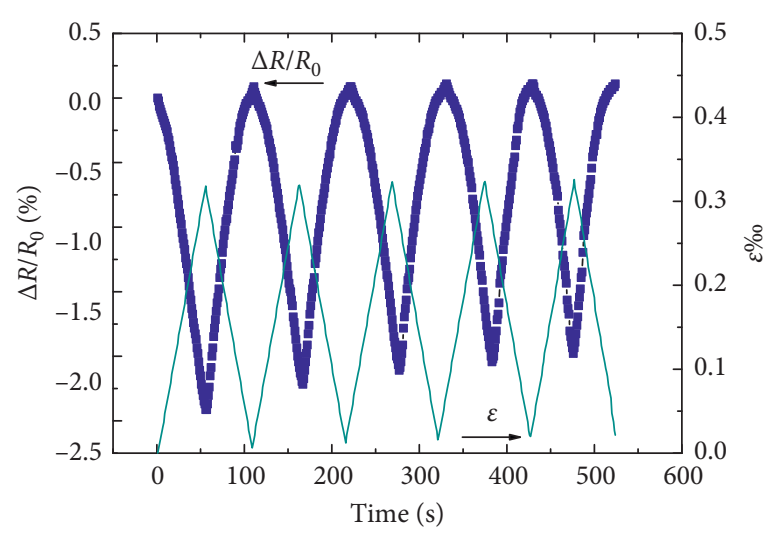

(b)

Figure 4: Piezoresistive behavior for test 1: (a) CM $\left(R_{0}=4801 \Omega\right)$; (b) WCM3 $\left(R_{0}=4600 \Omega\right)$.

of both ions and electrons. In case of high moisture, ionic conduction plays a dominant role for the piezoresistive behavior, while the transmission of electrons through MWCNTs reduces the piezoresistive sensitivity, which is reflected by the fact that the amplitude of $\Delta R / R_{0}$ for WCM3 is smaller than one for CM in each cycle of loading.

Ionic conduction is affected by the connection of pore water. During loading, the pore water may connect under compression and form pathways of ionic conduction, resulting in the decrease of resistance. During unloading, the pathways formed will break, and resistance increases. However, this process is not reversible because cement matrix may become wet as pore water flows by. As a result, the piezoresistive sensitivity decreases. As shown in Figure 4, the amplitude of $\Delta R / R_{0}$ becomes smaller and smaller with cyclic compression going on.

Figure 5 shows the piezoresistive behaviors of both CM and WCM3 for the second test. In this test, moisture content reduced about $0.75 \%$ for $\mathrm{CM}$ and $1.12 \%$ for $\mathrm{WCM} 3$, respectively. Due to this reduction, the resistance of specimens increased. Surprisingly, both CM and WCM3 presented an opposite piezoresistive response as test 1 . It is observed that, in each cycle, the value of $\Delta R / R_{0}$ increased monotonically upon the compressive loading, whiledecreased monotonically upon unloading, exhibiting negative piezoresistivity.

Furthermore, the amplitude of $\Delta R / R_{0}$ for CM decreased from about $5.5 \%$ to about $4 \%$. This indicates that moisture content may affect the piezoresistive sensitivity. With the reduction of moisture content, CM becomes less sensitive to strain. However, the addition of MWCNTs enhances the piezoresistive sensitivity. In this case of moisture content, the amplitude of $\Delta R / R_{0}$ for WCM3 reached a value of about 6 , greater than for CM.

Figures 6-8 illustrate the piezoresistive behaviors of both CM and WCM3 for the next three tests. With the further reduction of moisture content, the resistance of CM gradually increased. Before test 5 , the initial resistance became $603500 \Omega$, about 125 times higher than one before the first test. Accordingly, we believed that the conductivity of cementitious composites was greatly affected by the volume of porosity and moisture content in the composites. More specifically, decreasing the amount of water in the composites resulted in the increase of electric resistance, and vice versa. From all the tests, it was observed that the addition of MWCNTs reduced the resistance of cementitious materials.

Again, the piezoresistive responses of both $C M$ and WCM3 turned to be positive. The value of $\Delta R / R_{0}$ decreased monotonically upon the compressive loading, while it increased monotonically upon unloading. In these tests, the amplitude of $\Delta R / R_{0}$ for CM remained almost unchanged with the decrease of moisture content, being about $4 \%$. But, the amplitude of $\Delta R / R_{0}$ for WCM3 increased from $5 \%$ to $6 \%$, obviously greater than one for CM during each cyclic loading. This clearly shows that the piezoresistive responses of cementitious composites can be induced by the ionic conduction through pore fluids, while the addition of MWCNTs enhances the responses.

From these results, we believe that the variation of moisture content is one main reason for the change of piezoresistivity. In fact, there exist two conductive networks in cementitious composites. Network 1, being responsible for the ionic conduction, consists of capillary pores or voids that are filled completely or partially with water. These pores or voids may interconnect each other [26]. Network 2, being responsible for the electronic conduction, consists of connected MWCNTs or partially connected MWCNTs that are spaced by voids or matrix. Obviously, Network 1 depends on moisture content and the connection of capillary pores or voids, while Network 2 is related to moisture content and the connection of MWCNTs.

This can be further explained with the schematic illustration in Figure 9. Each network has three different conductive pathways. Figure 9(a) shows the case of high moisture content. In this figure, CR1 and CR2 refer to parallel pathways of pores or voids filled completely with water and are connected through transverse voids. CR3 refers to the pathway partially with water. MWCNTs may also form three conductive pathways, completely connected (CR4), partially connected (CR5, spaced by voids), and partially connected (CR6, spaced by matrix). Due to drying, moisture content will reduce. As a result, the connected pathways CR1 and CR2 will become less (Figure 9(b)) or 


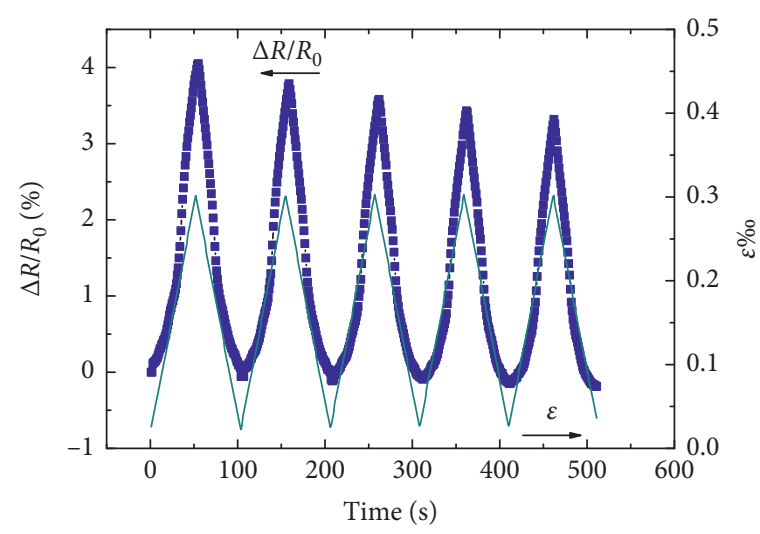

(a)

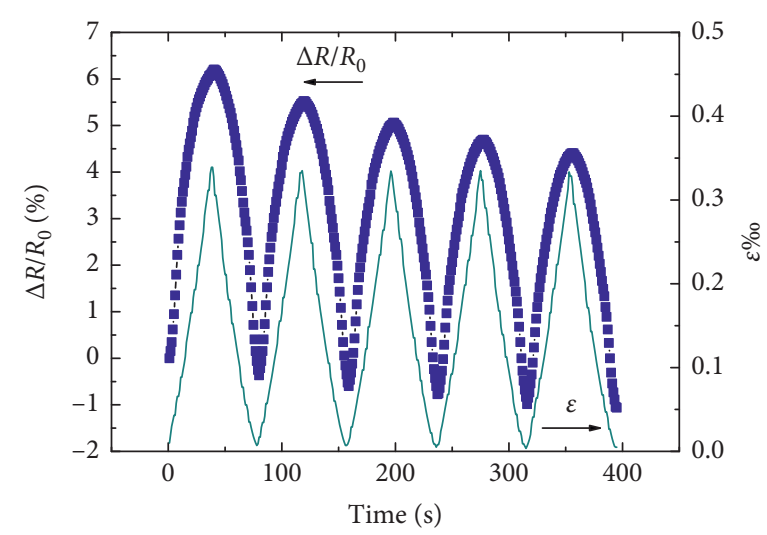

(b)

Figure 5: Piezoresistive behavior for test 2: (a) CM $\left(R_{0}=5333 \Omega\right)$; (b) WCM3 $\left(R_{0}=5079 \Omega\right)$.

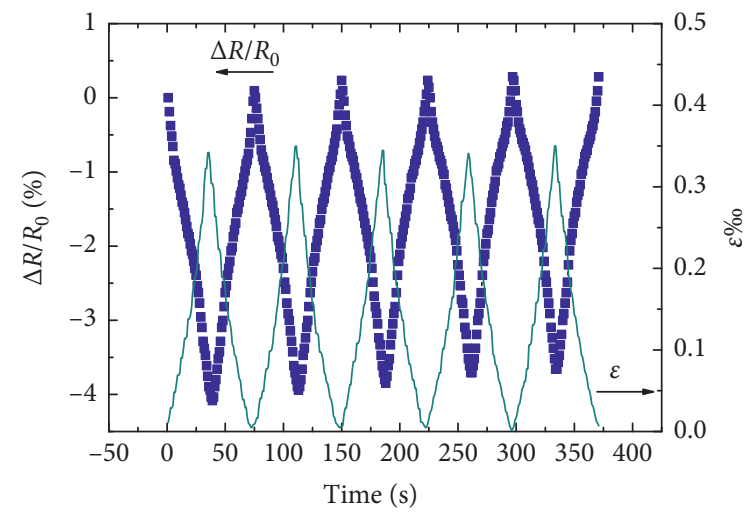

(a)

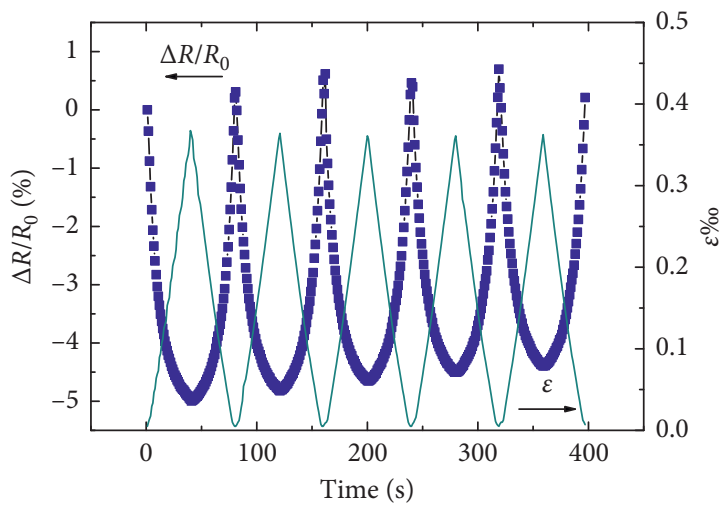

(b)

Figure 6: Piezoresistive behavior for test 3: (a) CM $\left(R_{0}=21310 \Omega\right)$; (b) WCM3 $\left(R_{0}=19477 \Omega\right)$.

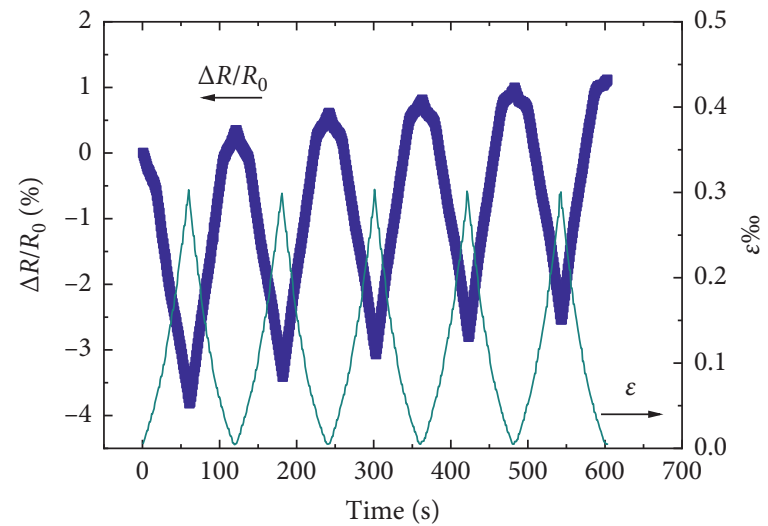

(a)

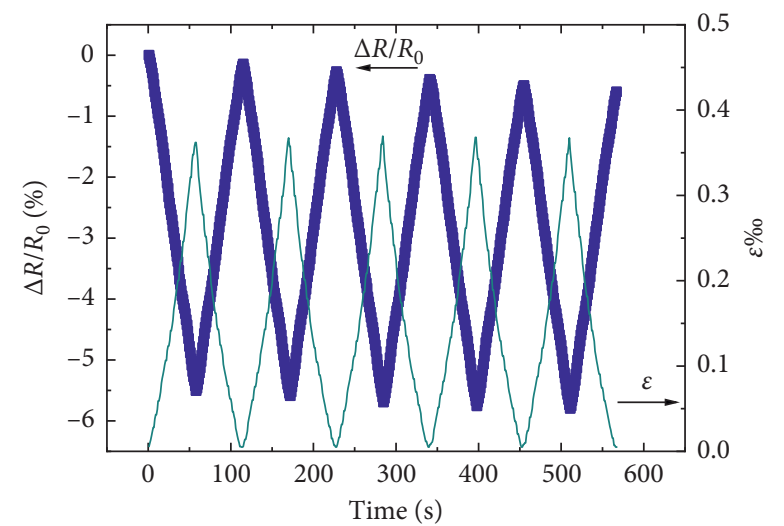

(b)

Figure 7: Piezoresistive behavior for test 4: (a) CM $\left(R_{0}=49300 \Omega\right)$; (b) WCM3 $\left(R_{0}=48140 \Omega\right)$.

partially connected (Figure 9(c)), leading to the reduction of ionic conduction. Moreover, drying process will increase the potential barrier between MWCNTs and reduce the tunneling effect of electrons. The electronic conduction through CR5 and CR6 reduces. Under compression, since both CR3 and CR5 may become connected, the ionic conduction through CR3 and the electronic conduction through CR5 increase. At the same time, the tunneling effect through CR6 becomes higher with the deformation of matrix. In all these processes, since CR4 is completely connected, the current through CR4 does not change, and therefore CR4 will not contribute to piezoresistivity. 


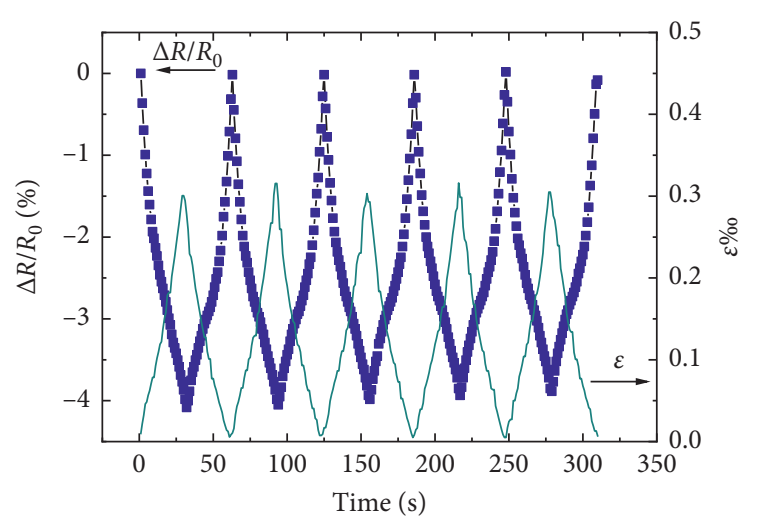

(a)

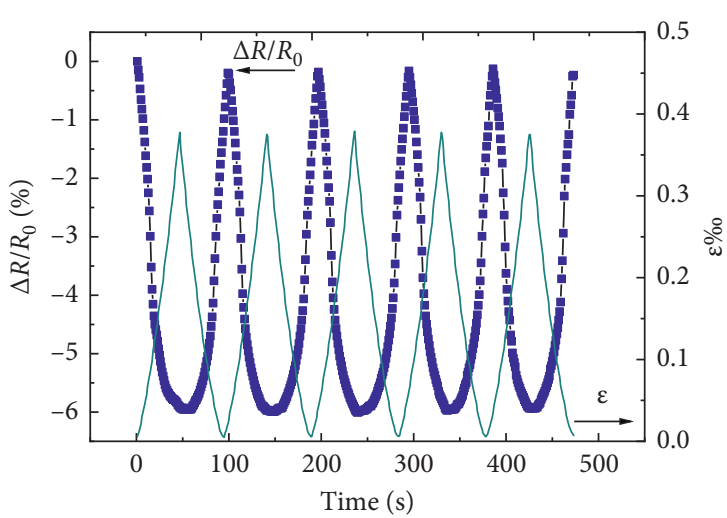

(b)

Figure 8: Piezoresistive behavior for test 5: (a) CM $\left(R_{0}=603500 \Omega\right)$; (b) WCM3 $\left(R_{0}=551200 \Omega\right)$.

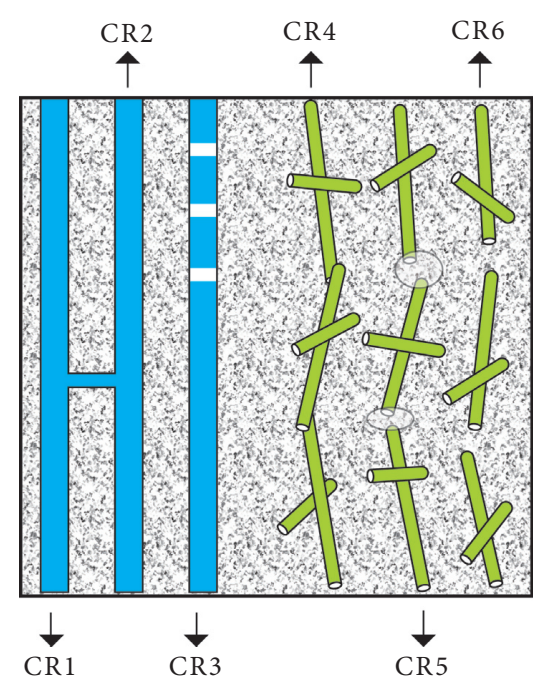

(a)

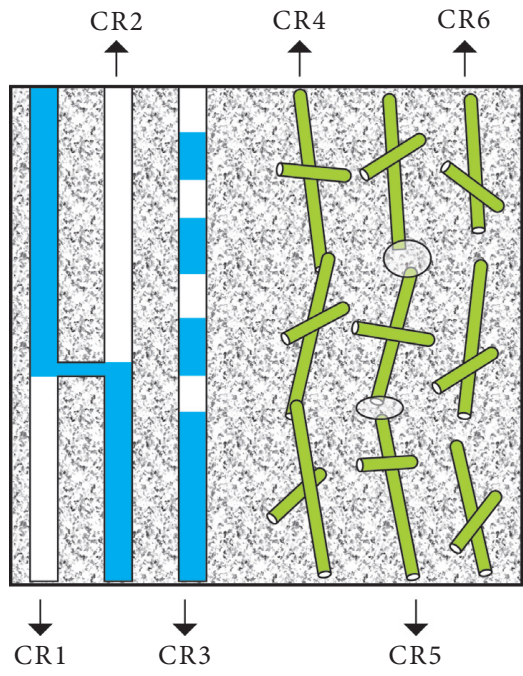

(b)

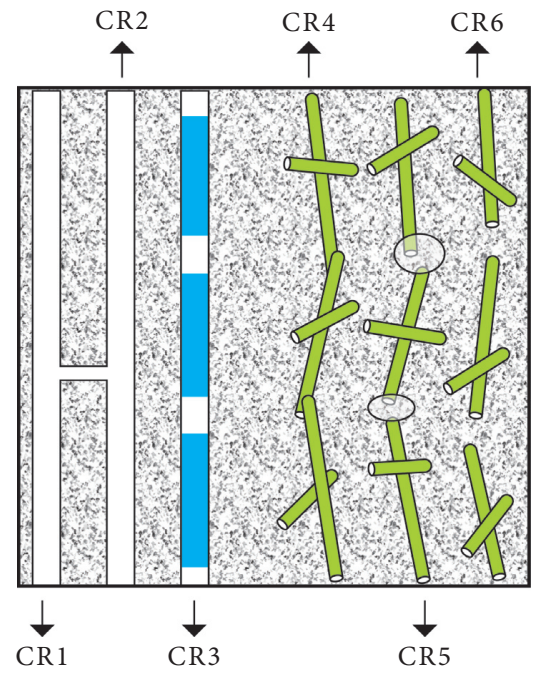

(c)

Figure 9: Schematic views of conductive pathways.

Suppose that $I_{1}^{0}$ is the current through Network 1 and $I_{2}^{0}$ is the current through Network 2 before loading. Under compression, the composites will deform, and the current through the two networks becomes $I_{1}$ and $I_{2}$, respectively. We have the increments $\Delta I_{1}=I_{1}-I_{1}^{0}$ and $\Delta I_{2}=I_{2}-I_{2}^{0}$. The total current increment through the composites is that $\Delta I=\Delta I_{1}+\Delta I_{2}$. Since both $\Delta I_{1}$ and $\Delta I_{2}$ are either positive or negative, it is probable that the following cases will appear:

Case 1. $\Delta I_{1}>0 ; \Delta I_{2}>0$. It is definite that $\Delta I>0$. Consequently, $\Delta R / R_{0}<0$. The piezoresistivity is positive. Moreover, the addition of MWCNTs enhances the piezoresistive sensitivity.

Case 2. $\Delta I_{1}>0 ; \Delta I_{2}<0$. If $\left|\Delta I_{1}\right|>\left|\Delta I_{2}\right|$, then $\Delta I>0$. We still have $\Delta R / R_{0}<0$. The piezoresistivity is positive. However, the ionic conduction plays a main role, and the addition of MWCNTs reduces the piezoresistive sensitivity.

Case 3. $\Delta I_{1}>0 ; \Delta I_{2}<0$. If $\left|\Delta I_{1}\right|<\left|\Delta I_{2}\right|$, then $\Delta I<0$. In this case, $\Delta R / R_{0}>0$. The piezoresistivity is negative, and the electronic conduction through MWCNTs plays a main role for the piezoresistive response.

Case 4. $\Delta I_{1}<0 ; \Delta I_{2}>0$. If $\left|\Delta I_{1}\right|>\left|\Delta I_{2}\right|$, then $\Delta I<0$. As a result, $\Delta R / R_{0}>0$. The piezoresistivity is negative.

Case 5. $\Delta I_{1}<0 ; \Delta I_{2}>0$. If $\left|\Delta I_{1}\right|<\left|\Delta I_{2}\right|$, then $\Delta I>0$. We have $\Delta R / R_{0}<0$. The piezoresistivity is positive.

Case 6. $\Delta I_{1}<0 ; \Delta I_{2}<0$. Then $\Delta I<0$. In this case, $\Delta R / R_{0}>0$. The piezoresistivity is negative.

For a definite specimen, both the connection of capillary pores or voids and the connection of MWCNTs are fixed. Whether $\Delta I_{1}$ and $\Delta I_{2}$ are positive or negative depends on the moisture content in the material. It is seen that tunneling effect occurs through CR3 in both Figures 9(a) and 9(c), and current increases during compression. As a result, $\Delta I_{1}>0$. However, in Figure 9(b), even if the tunneling current through CR3 increases, the bridge between CR1 and CR2 may be narrowed or blocked under compression, a phenomenon called "bottleneck effect" [26], which leads to the 
decrease of total current. Therefore, $\Delta I_{1}<0$. Similarly, when voids are full of water in case of higher moisture content (Figures 9(a) and 9(b)), CR5 is conductively connected. Due to compressive loading, the conductive area may decrease and then $\Delta I_{2}<0$. Otherwise, CR5 becomes disconnected (Figure $9(\mathrm{c})$ ). In this case, $\Delta I_{2}>0$, since the tunneling current through CR5 increases.

Based on the analysis, we infer that test 1 (Figure 4) belongs to Case 2, test 2 (Figure 5) is Case 6, and the next three tests (Figures 6-8) are Case 1.

\section{Theoretical Modelling of Piezoresistivity}

As discussed in Section 3, both ionic conduction and electronic conduction may happen in the cement matrix composites containing MWCNTs. The ionic conduction is induced due to the existence of pore solutions in the composites, while the electronic conduction occurs through connected or adjacent MWCNTs. Therefore, the composite conductivity can be expressed as

$$
\sigma=\sigma_{1}+\sigma_{2},
$$

where $\sigma, \sigma_{1}$, and $\sigma_{2}$ represent the composite conductivity, ionic conductivity, and electronic conductivity, respectively. According to Archie's law [29],

$$
\sigma_{1}=\sigma_{w} \phi^{\beta},
$$

where $\sigma_{w}$ is the electrical conductivity of pore solution and $\phi$ is the composite porosity. The parameter $\beta$ reflects the connectivity of pore solution, which depends on the moisture content and pore structure, as well as the strain during loading and unloading. Based on the tunneling theory $[28,30]$, it is supposed to be

$$
\beta=\beta_{0} \exp (-a \cdot \varepsilon)+1,
$$

where $\beta_{0}$ and $a$ are parameters remaining to be experimentally determined.

The electronic conductivity $\sigma_{2}$ is attributed to the bridging conductivity of MWCNTs and the tunneling effect between adjacent MWCNTs. It should take a similar form as equation (3):

$$
\sigma_{2}=\sigma_{f} \cdot \phi_{f}^{\gamma},
$$

where $\sigma_{f}$ is the MWCNTs conductivity, $\phi_{f}$ is the volume fraction of MWCNTs, and $\gamma$ reflects the tunneling effect of MWCNTs in the composites. In the same way as $\beta$, it is assumed that

$$
\gamma=\gamma_{0} \exp (-b \cdot \varepsilon)+1,
$$

where $\gamma_{0}$ and $b$ are parameters remaining to be experimentally determined.

Then, we have

$$
\sigma=\sigma_{1}+\sigma_{2}=\sigma_{w} \phi^{\beta}+\sigma_{f} \phi_{f}^{\gamma} .
$$

It is noted that, in the case without MWCNTs, $\sigma=\sigma_{1}=\sigma_{w} \phi^{\beta}$. If the composites are dry enough, the conductivity due to pore fluids can be ignored and the composite conductivity may be simplified to $\sigma=\sigma_{2}=\sigma_{f} \phi_{f}^{\gamma}$.

The resistance of specimens can be calculated by

$$
R=\frac{\rho L}{A},
$$

where $L$ is the length between two inner electrical contacts, $A$ is the embedded area of electrical contact in the composites, and $\rho$ is the resistivity of composites. The piezoresistivity defined by equation (1) becomes

$$
\frac{\Delta R}{R_{0}}=\frac{\rho L S_{0}}{\rho_{0} L_{0} S}-1
$$

where $\rho_{0}, L_{0}$, and $S_{0}$ are the initial value prior to any loading. Under the condition of uniaxial compression and with $\rho=1 / \sigma$,

$$
\frac{\Delta R}{R_{0}}=\frac{\sigma_{0}\left(1+\varepsilon_{z}\right)}{\sigma\left(1-\nu \varepsilon_{z}\right)^{2}}-1,
$$

where $\varepsilon_{z}$ is the longitudinal strain and $\sigma_{0}$ is the initial value of composite conductivity. By using equation (7), we have

$$
\frac{\Delta R}{R_{0}}=\frac{\sigma_{w} \phi_{0}^{\beta_{0}+1}+\sigma_{f} \phi_{f}^{\gamma_{0}+1}}{\sigma_{w} \phi^{\beta}+\sigma_{f} \phi_{f}^{\gamma}} \frac{\left(1+\varepsilon_{z}\right)}{\left(1-\nu \varepsilon_{z}\right)^{2}}-1 .
$$

The porosity of composites will vary under loading and can be calculated by [31]

$\phi=\frac{\phi_{0}}{1-(1-2 v) \varepsilon_{z}}\left[1-\frac{\left(4 \mu_{m}+3 k_{m}\right)\left(1-\sqrt[3]{1-(1-2 v) \varepsilon_{z}}\right)}{4 \mu_{m}+3 k_{m} \phi_{0}}\right]^{3}$,

where $\phi_{0}$ is the initial porosity of composites and $\mu_{m}$ and $k_{m}$ are the shear modulus and the bulk modulus, respectively.

From equation (11), we can see that the piezoresistivity depends on the porosity of composites, the volume fraction of MWCTNs, and the connectivity parameters. Once these parameters are defined, the piezoresistive behavior with strain is determined. However, it is not easy to determine the connectivity parameters $\beta_{0}, \gamma_{0}, a$, and $b$. Here, we approximate them according to the following two steps.

Step 1. Consider the composite without MWCNTs. In this case, $\sigma=\sigma_{1}=\sigma_{w} \phi^{\beta}$. From the theory of four-probe method [32],

$$
\sigma=\frac{1}{F_{1} \cdot F_{2} \cdot C \cdot R}
$$

where $C$ is a parameter relating to the positions of four probes and $F_{1}$ and $F_{2}$ are geometric corrections of a specimen. For the specimen shown in Figure 1, $C=8.373, F_{1}=1.0$, and $F_{2}=0.9895$. Prior to loading, $\varepsilon_{z}=0$. Then, $R_{0} \longrightarrow \sigma_{0} \longrightarrow \beta_{0}$.

Choosing a pair of measured resistance $R$ and strain $\varepsilon_{z}$ at the beginning of loading, we have $R \longrightarrow \sigma \longrightarrow a$ (or multiple pairs of measured resistance $R$ and strain $\varepsilon_{z}$, and calculate the average of $a$ ). 


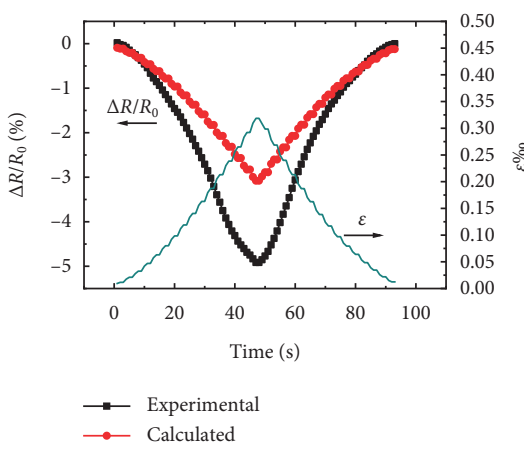

(a)

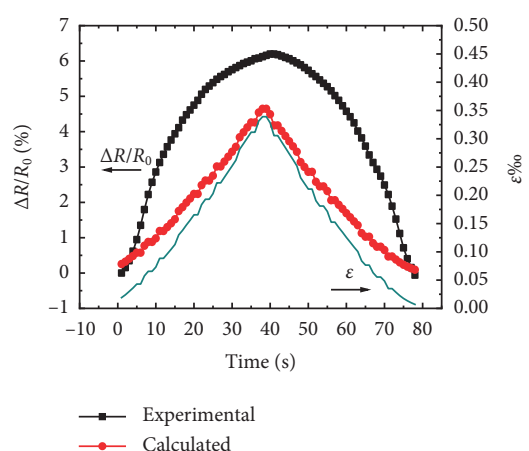

(d)

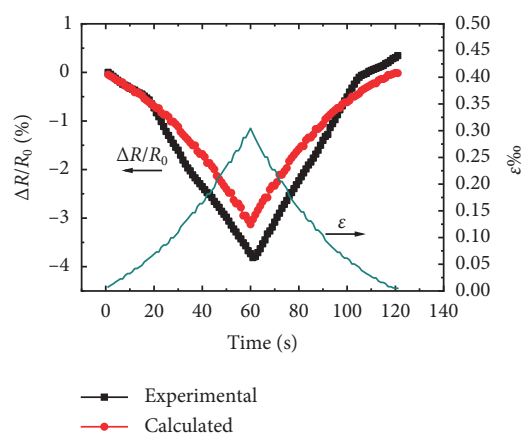

(g)

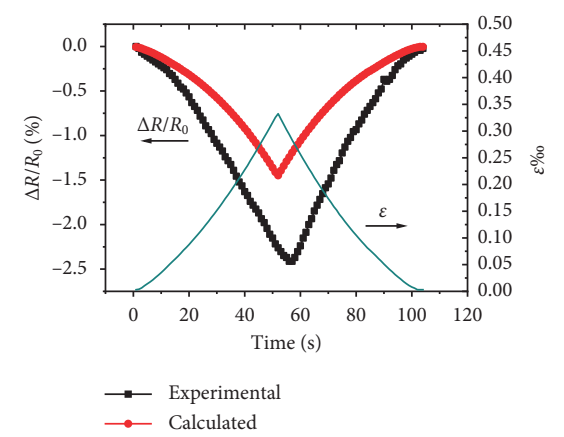

(b)

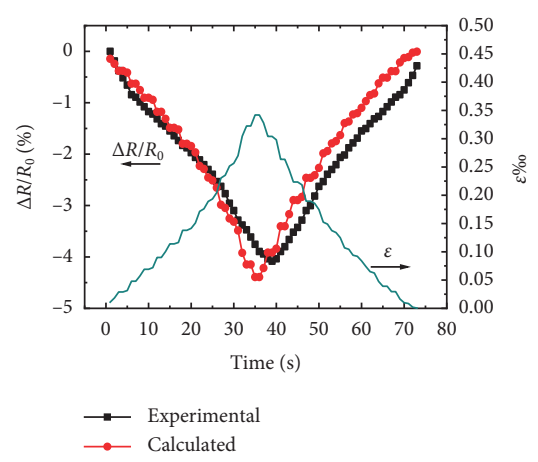

(e)

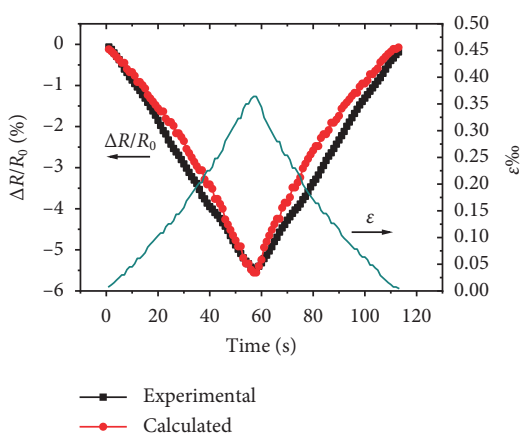

(h)

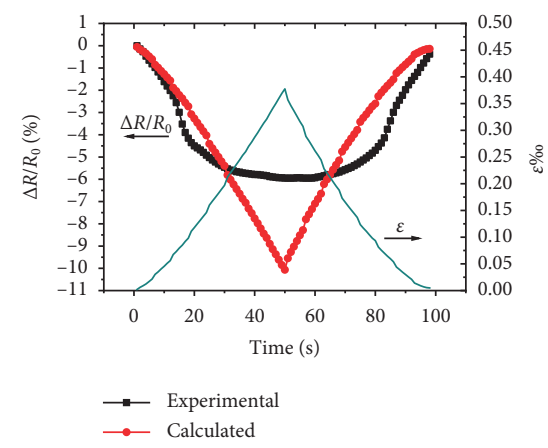

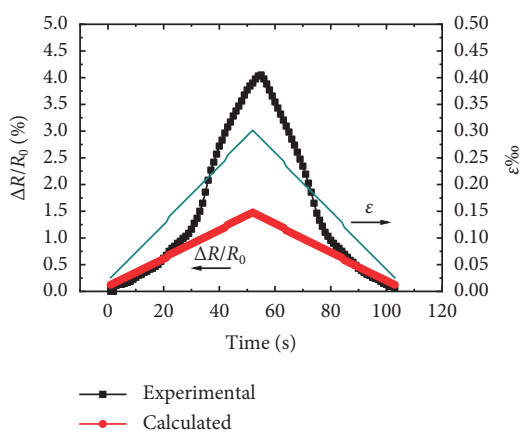

(c)

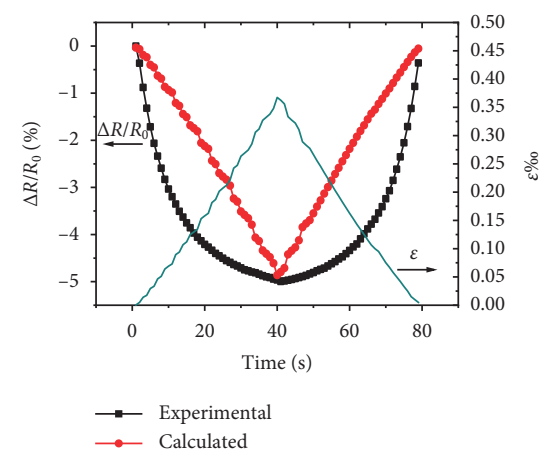

(f)

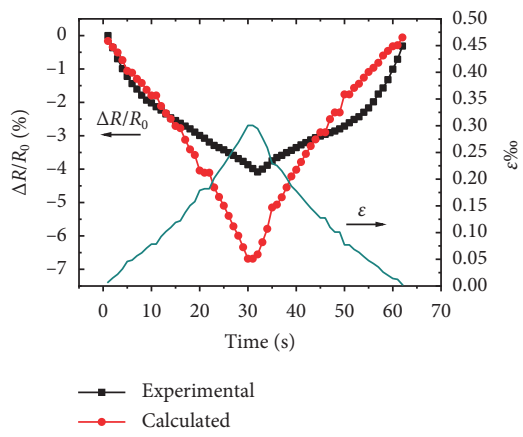

(i)

(j)

Figure 10: The calculated piezoresistivity of CM (a, c, e, g, i) and WCM3 (b, d, f, h, j).

Step 2. Consider the composite with MWCNTs. The composite conductivity is shown in equation (7). In the equation, $\beta_{0}$ and $a$ are assumed approximately to be the same as step one. Since $\varepsilon_{z}=0$ before loading, $R_{0} \longrightarrow \sigma_{0} \longrightarrow \gamma_{0}$. Choosing a pair of measured resistance $R$ and strain $\varepsilon_{z}$ at the beginning of loading,
$R \longrightarrow \sigma \longrightarrow b$ (or multiple pairs of measured resistance $R$ and strain $\varepsilon_{z}$, and calculate the average of $b$ ).

Using the above formulas, we calculated the piezoresistive responses of specimens CM and WCM3, with the elastic modulus of composites being $30 \mathrm{GPa}$ and the Poisson 
ratio being 0.21. Moreover, $\phi_{f}=0.00115, \sigma_{f}=100 \mathrm{~s} / \mathrm{cm}$, $\phi_{0}=0.28$, and $\sigma_{w}=0.1 \mathrm{~s} / \mathrm{cm}[33]$. The results for the first cycle of loading are shown in Figure 10.

It is seen thatin case of moderate moisture content (test 3 and test 4), the calculated piezoresistive responses of both CM and WCM3 agree well with the testing results (Figures 10(e)-10(h)), indicating that this theoretical model can predict reasonably the piezoresistivity of cementitious composites incorporating MWCNTs. Accordingly, it further shows that the piezoresistive responses of the composites are induced by not only the electronic conduction occurring through MWCNTs, but also the ionic conduction due to pore fluids in the composites. The effect of moisture content cannot be ignored.

However, when moisture content is high or low, the discrepancy between the calculated responses and the experimental data appears and becomes obvious with the increase of loading.

\section{Conclusions}

An experimental research was performed to investigate the piezoresistivity of cementitious composites incorporating MWCNTs due to the variation of moisture content. The variation of moisture content was controlled by drying the specimens in an oven, and the weight loss of these specimens was used as an index. Both positive and negative piezoresistivity were observed during the process of cyclic compression. In most cases of moisture content, the fractional change in resistance $\Delta R / R_{0}$ decreased monotonically upon compressive loading and increased monotonically upon unloading, exhibiting positive piezoresistivity. When moisture content was in a specific range, the fractional change in resistance $\Delta R / R_{0}$ increased monotonically upon compressive loading and decreased monotonically upon unloading, demonstrating negative piezoresistivity. The whole transition process from positive piezoresistivity to negative piezoresistivity and then positive piezoresistivity was presented. Moreover, it was found that the amplitude of piezoresistivity changed due to variation of moisture content. The mechanism for these phenomena is discussed.

A theoretical model of piezoresistivity, including both ionic conduction and electronic conduction, is proposed. This model is able to predict the effects of porosity, the volume fraction of MWCTNs, and the connectivity parameters on the piezoresistivity. Numerical results show that, in case of moderate moisture content, the calculated piezoresistive responses of specimens agree well with the testing data. However, when moisture content is high or low, discrepancy appears and becomes obvious with the increase of loading. In future work, a more reasonable model should be developed to overcome these drawbacks. Possible applications of piezoresistivity to practical structures for strain sensing should also be studied.

\section{Data Availability}

The data used to support the findings of this study are available from the corresponding author upon request.

\section{Conflicts of Interest}

The authors declare that they have no conflicts of interest.

\section{Acknowledgments}

The authors gratefully acknowledge the funding provided by Key Laboratory Project of Guangdong Higher Education Institutes (2013CXZDA017) and Key Laboratory of Structure and Wind Tunnel of Guangdong Higher Education Institutes (201803).

\section{References}

[1] F. Sanchez and K. Sobolev, "Nanotechnology in concrete-a review," Construction and Building Materials, vol. 24, no. 11, pp. 2060-2071, 2010.

[2] F. Pacheco-Torgal and S. Jalali, "Nanotechnology: advantages and drawbacks in the field of construction and building materials," Construction and Building Materials, vol. 25, no. 2, pp. 582-590, 2011.

[3] M. Yu, O. Lourie, M. J. D. K. Moloni, T. F. Kelly, and R. S. Ruoff, "Strength and breaking mechanism of multiwalled carbon nanotubes under tensile load," Science, vol. 287, no. 5453, pp. 637-640, 2000.

[4] M. Rafiee, J. Yang, and S. Kitipornchai, "Thermal bifurcation buckling of piezoelectric carbon nanotube reinforced composite beams," Computers \& Mathematics with Applications, vol. 66, no. 7, pp. 1147-1160, 2013.

[5] M. Rafiee, F. Nitzsche, and M. R. Labrosse, "Cross-sectional design and analysis of multiscale carbon nanotubes-reinforced composite beams and blades," International Journal of Applied Mechanics, vol. 10, no. 3, p. 1850032, 2018.

[6] E. W. Wong, P. E. Sheehan, and C. M. Lieber, "Nanobeam mechanics: elasticity, strength, and toughness of nanorods and nanotubes," Science, vol. 277, no. 5334, pp. 1971-1975, 1997.

[7] G. Y. Li, P. M. Wang, and X. Zhao, "Mechanical behavior and microstructure of cement composites incorporating surfacetreated multi-walled carbon nanotubes," Carbon, vol. 43, no. 6, pp. 1239-1245, 2005.

[8] A. P. Singh, B. K. Gupta, M. Mishra et al., "Multiwalled carbon nanotube/cement composites with exceptional electromagnetic interference shielding properties," Carbon, vol. 56, pp. 86-96, 2013.

[9] H. K. Kim, I. W. Nam, and H. K. Lee, "Enhanced effect of carbon nanotube on mechanical and electrical properties of cement composites by incorporation of silica fume," Composite Structures, vol. 107, pp. 60-69, 2014.

[10] S. Xu, J. Liu, and Q. Li, "Mechanical properties and microstructure of multi-walled carbon nanotube-reinforced cement paste," Construction and Building Materials, vol. 76, pp. 1623, 2015.

[11] A. Tamimi, N. M. Hassan, K. Fattah, and A. Talachi, "Performance of cementitious materials produced by incorporating surface treated multiwall carbon nanotubes and silica fume," Construction and Building Materials, vol. 114, pp. 934-945, 2016.

[12] M. D. C. Camacho, O. Galao, F. J. Baeza, E. Zornoza, and P. Garcés, "Mechanical properties and durability of CNT cement composites," Materials, vol. 7, no. 3, pp. 1640-1651, 2014.

[13] Y. F. Wang, H. Hu, and C. Q. Rong, "The effect of the Diameter of carbon nanotube on the mechanical and electrical 
properties of cement mortar," Key Engineering Materials, vol. 730, pp. 479-485, 2017.

[14] P. Xie, Z. Wang, Z. Zhang et al., "Silica microsphere templated self-assembly of a three-dimensional carbon network with stable radio-frequency negative permittivity and low dielectric loss," Journal of Materials Chemistry C, vol. 6, no. 19, pp. 5239-5249, 2018.

[15] P. Xie, W. Sun, Y. Liu et al., "Carbon aerogels towards new Candidates for double negative metamaterials of low density," Carbon, vol. 129, pp. 598-606, 2018.

[16] Y. Qu, Y. Du, G. Fan et al., "Low-temperature sintering graphene/ $\mathrm{CaCu}_{3} \mathrm{Ti}_{4} \mathrm{O}_{12}$ nanocomposites with tunable negative permittivity," Journal of Alloys and Compounds, vol. 771, pp. 699-710, 2019.

[17] P. T. Xie, Z. D. Zhang, Z. Y. Wang, K. Sun, and R. H. Fan, "Targeted double negative properties in silver/silica random metamaterials by precise control of Microstructures," Research, vol. 2019, pp. 1-11, 2019.

[18] G. Y. Li, P. M. Wang, and X. Zhao, "Pressure-sensitive properties and microstructure of carbon nanotube reinforced cement composites," Cement and Concrete Composites, vol. 29 , no. 5, pp. 377-382, 2007.

[19] M. Saafi, "Wireless and embedded carbon nanotube networks for damage detection in concrete structures," Nanotechnology, vol. 20, no. 39, pp. 1-7, 2009.

[20] B. Han, X. Yu, and E. Kwon, "A self-sensing carbon nanotube/ cement composites for traffic monitoring," Nanotechnology, vol. 20, no. 44, pp. 445-501, 2009.

[21] B. Han, X. Yu, K. Zhang, E. Kwon, and J. Ou, "Sensing properties of CNT-filled cement-based stress sensors," Journal of Civil Structural Health Monitoring, vol. 1, no. 1-2, pp. 17-24, 2011.

[22] X. Yu and E. Kwon, "A carbon nanotube/cement composite with piezoresistive properties," Smart Materials and Structures, vol. 18, no. 5, Article ID 055010, 2009.

[23] M. S. Konsta-Gdoutos and C. A. Aza, "Self sensing carbon nanotube (CNT) and nanofiber (CNF) cementitious composites for real time damage assessment in smart structures," Cement and Concrete Composites, vol. 53, pp. 162-169, 2014.

[24] B. Han, X. Yu, and J. Ou, "Effect of water content on the piezoresistivity of MWNT/cement composites," Journal of Materials Science, vol. 45, no. 14, pp. 3714-3719, 2010.

[25] H. K. Kim, I. S. Park, and H. K. Lee, "Improved piezoresistive sensitivity and stability of CNT/cement mortar composites with low water-binder ratio," Composite Structures, vol. 116, pp. 713-719, 2014.

[26] D. Sohn and T. O. Mason, "Electrically induced microstructural changes in Portland cement pastes," Advanced Cement Based Materials, vol. 7, no. 3-4, pp. 81-88, 1998.

[27] S. Frank, P. Poncharal, Z. L. Wang, and W. A. de Heer, "Carbon nanotube quantum resistors," Science, vol. 280, no. 5370 , pp. $1744-1746,1998$.

[28] J. Zhao, C. Buia, J. Han, and J. P. Lu, "Quantum transport properties of ultrathin silver nanowires," Nanotechnology, vol. 14, no. 5, pp. 501-504, 2002.

[29] P. W. J. Glover, M. J. Hole, and J. Pous, “A modified Archie's law for two conducting phases," Earth and Planetary Science Letters, vol. 180, no. 3-4, pp. 369-383, 2000.

[30] W. S. Bao, S. A. Meguid, Z. H. Zhu, and G. J. Weng, "Tunneling resistance and its effect on the electrical conductivity of carbon nanotube nanocomposites," Journal of Applied Physics, vol. 111, Article ID 093726, 2012.
[31] J. Liu and X. L. Du, "Variation of porosity and its effect on the deformation process of concrete," Journal of Engineering Mechanics, vol. 30, no. 6, pp. 183-190, 2013.

[32] Y. C. Sun, Z. S. Fan, X. Y. Sun, and Q. F. Ning, "Relationship between geometric effect correction on resistivity measurement methods," Semiconductor Science and Technology, vol. 25 , pp. $38-41,2000$.

[33] F. Rajabipour and J. Weiss, "Electrical conductivity of drying cement paste," Materials and Structures, vol. 40, no. 10, pp. 1143-1160, 2007. 